\title{
Inelastic decay of electrons in Shockley-type metal-organic interface states
}

\author{
S. S. Tsirkin, ${ }^{1,2,3}$ N. L. Zaitsev, ${ }^{4}$ I. A. Nechaev,${ }^{1,2,3,5}$ R. Tonner ${ }^{4}{ }^{4}$ U. Höfer, ${ }^{1,4}$ and E. V. Chulkov ${ }^{1,2,3,5,6}$ \\ ${ }^{1}$ Donostia International Physics Center (DIPC), 20018 San Sebastián/Donostia, Basque Country, Spain \\ ${ }^{2}$ Tomsk State University, 634050, Tomsk, Russia \\ ${ }^{3}$ Saint Petersburg State University, Saint Petersburg, 198504, Russia \\ ${ }^{4}$ Philipps-Universität Marburg, D-35032, Marburg, Germany \\ ${ }^{5}$ Centro de Física de Materiales CFM - MPC, Centro Mixto CSIC-UPV/EHU, 20080 San Sebastián/Donostia, Basque Country, Spain \\ ${ }^{6}$ Departamento de Física de Materiales UPV/EHU, Facultad de Ciencias Químicas, UPV/EHU, Apdo. 1072, 20080 San Sebastián/Donostia, \\ Basque Country, Spain
}

(Received 28 July 2015; published 18 December 2015)

\begin{abstract}
We present a theoretical study of lifetimes of interface states (IS) on metal-organic interfaces PTCDA/Ag(111), NTCDA/Ag(111), PFP/Ag(111), and PTCDA/Ag(100), describing and explaining the recent experimental data. By means of unfolding the band structure of one of the interfaces under study onto the $\mathrm{Ag}(111)$ Brillouin zone we demonstrate that the Brillouin zone folding upon organic monolayer deposition plays a minor role in the phase space for electron decay, and hence weakly affects the resulting lifetimes. The presence of the unoccupied molecular states below the IS gives a small contribution to the IS decay rate, which is mostly determined by the change of the phase space of bulk states upon the energy shift of the IS. The calculated lifetimes follow the experimentally observed trends. In particular, we explain the trend of the unusual increase of the IS lifetimes with rising temperature.
\end{abstract}

DOI: 10.1103/PhysRevB.92.235434

PACS number(s): 73.20.-r, 79.60.Dp, 78.47.-p

\section{INTRODUCTION}

Interfaces between organic thin films grown on single crystal metals serve as structurally well-defined models for contacts in organic semiconductor devices [1-3]. The efficiency of the charge transfer across such interfaces depends on the alignment of the molecular levels with respect to the Fermi level of the metal and the overlap of the wave functions of the metallic and molecular states. An important additional factor is the possible existence of new interface electronic states. Indeed, many well-defined interfaces between organic semiconductors and metals exhibit such interface-specific states that exist independently of the detailed moleculesubstrate interaction [4-7]. Like the surface states (SSs) of clean metals they are a consequence of the breaking of translational symmetry perpendicular to the interface. In the Shockley-type interface states (ISs) that have been identified at $\operatorname{Ag}(111)$ and $\operatorname{Ag}(100)$ interfaces the electrons are able to move almost freely parallel to the interface, whereas the local charge density in the vicinity of the first molecular layer is strongly corrugated and resembles that of molecular orbitals. These general properties of the states have been revealed in recent experimental and theoretical works [8-19]. For a few systems also the important factors that determine their electronic structure could be investigated [16,19].

How big a role these interface states play in terms of the overall charge transfer is not yet clear. In order to clarify their role and to perhaps exploit their properties for the engineering of contacts, a good understanding of the electron dynamics of the states is indispensable. Two-photon photoemission (2PPE) experiments have measured lifetimes between 10 and $200 \mathrm{fs}$ for electrons excited into normally unoccupied interface states above the Fermi level $[9,16,18,19]$. From these short lifetimes a large overlap of the wave function with the metal has been concluded. These conclusions, although confirmed by density functional calculations $[12,13,16-18]$, are based on very simplistic assumptions on the nature of electronic decay processes at such an interface. Many-body calculations, such that exist for surfaces states of clean metal surfaces [20], have not been performed so far. Only with such realistic theoretical descriptions is it possible to interpret the measured lifetimes in terms of microscopic decay processes.

In this publication we make a first step in this direction. We perform a theoretical study of the decay of electrons in the ISs formed at the interfaces of silver with commensurate ordered monolayers of such organic molecules as perylene-3,4,9,10tetracarboxylic acid dianhydride (PTCDA), naphthalene1,4,5,8-tetracarboxylic acid dianhydride (NTCDA), and perfluoropentacene (PFP). On an equal footing we perform $a b$ initio density functional calculations of the electronic structure of all the studied interfaces. Our calculations show that the hybridization of molecular and metallic states is rather small in the region of the projected band gap of the metal. New elastic decay channels, which in principle could open up due to reduced translational symmetry of the organic overlayers, will thus only have a weak influence on the electron decay of the interface state. This allows us to focus on the inelastic decay of electrons excited to the IS. We calculate the corresponding lifetimes in the self-energy formalism of many-body theory using the $G W$ approximation [21]. In order to make the calculations for such large systems feasible, we use one-dimensional (1D) model potentials for an approximate description of the electronic structure of the interfaces. The potentials are based on the so-called Chulkov potential $[22,23]$ of clean surfaces. They are modified in order to reproduce the experimentally observed energy upshift of the Shockley surface state in the presence of the organic overlayers. Comparison with the $a b$ initio calculations allows us to judge how well the potentials reproduce the probability density of the states perpendicular to 
the interfaces. The results are in good overall agreement with the experimental data for PTCDA/Ag(111), NTCDA/Ag(111), PFP/Ag(111), and PTCDA/Ag(100). Calculated lifetimes are generally longer than experimental ones but agree well with experimental trends.

\section{AB INITIO CALCULATION OF ELECTRONIC STRUCTURE OF INTERFACES}

In order to have detailed information about the electronic structure of the interfaces under study, we performed $a b$ initio calculations within the periodic slab geometry. We used the OPENMX (version 3.7) code [24], which is based on density functional theory (DFT) and the linear combination of localized pseudoatomic orbital (LCPAO) method [25-27]. We applied the generalized gradient approximation (GGA) of Ref. [28] for the exchange-correlation functional. Also we exploited norm-conserving pseudopotentials [29] in order to replace deep core potentials by shallow ones. For silver atoms we set basis functions to Ag7.0-s $2 p 2 d 2 f 1$, while for hydrogen, carbon, and oxygen atoms we use H6.0-s $2 p 1$, C6.0-s $2 p 2 d 1$, and 06.0-s $2 p 2 d 1$, respectively. On the example of silver, this notation means that two primitive orbitals for each $s, p$, and $d$ orbital and one primitive orbital for the $f$ orbital were used for representation of the basis functions with the cutoff radius of 7.0 Bohrs.

To improve the description of the surface state (SS), we used the enlarged cutoff radius (9.0 Bohrs) for silver atoms in the uppermost layers of the slab. The latter contains ten silver layers together with the molecular monolayer (ML) attached on one side of the silver film. We use the experimental adsorption geometry (the molecules arrangement, surface unit cell, and vertical distance between the silver surface and carbon atoms of molecules) for NTCDA [30-33], PTCDA [34-36], and PFP [37-39] MLs on the Ag(111) surface, neglecting the bending of the molecules. The oxygen atoms of NTCDA and PTCDA monolayers on $\mathrm{Ag}(111)$ are fixed at the same distance as carbon ones. The optimized geometry of PTCDA/Ag(100) found in Ref. [18] is used in our calculation. The real-space grid for numerical integration and solution of the Poisson equation was specified by the energy cutoff of $250 \mathrm{Ry}$. The total-energy convergence was better than $0.027 \mathrm{meV}$. The surface Brillouin zone (SBZ) of the supercell was sampled with a $3 \times 3 \times 1$ mesh of $\mathbf{k}$ points. The following coordinates are used in the article: the $z$ axis is directed perpendicular to the surface outside the metal, the position of $z=0$ corresponding to the plane of the topmost Ag atomic layer on the side where the ML is attached. The $x-y$ plane is parallel to the surface.

As shown in Fig. 1, due to the reduced translational symmetry the unit cell of the interfaces is sufficiently larger than the $(1 \times 1)$ cell naturally used for the bare surfaces. Therefore, the SBZ becomes smaller, and, consequently, the metal bands of the initial SBZ corresponding to the $(1 \times 1)$ unit cell get folded into the reduced SBZ, hybridizing with the orbitals of the molecular ML [Figs. 2(a)-2(d)]. This leads to a surface band structure that does not exhibit the projected band gap at the $\bar{\Gamma}$ point any more. To restore the $(1 \times 1)$ unit-cell representation of the interface electronic structure, we performed an unfolding procedure using the BandUP code [40-42], based on the method by Popescu and

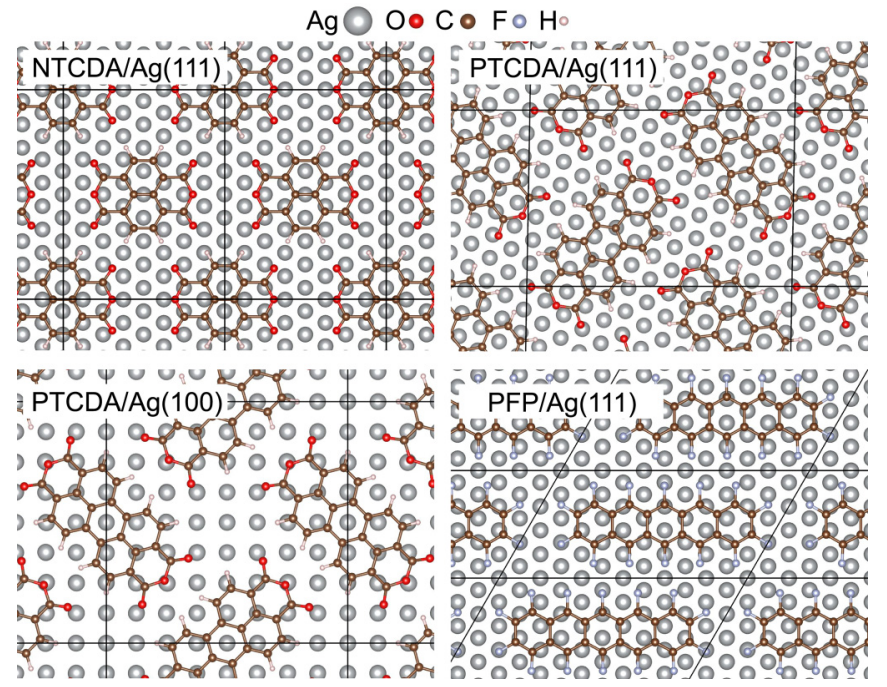

FIG. 1. (Color online) Unit cells of the interfaces under study (top view).

Zunger [43]. Upon the unfolding procedure each electronic state $m \mathbf{K}$ (where $m$ is the band index and $\mathbf{K}$ is the wave vector in the SBZ of the interface) is projected onto a set of corresponding points $\mathbf{k}_{i}$ in the unfolded Brillouin zone (UBZ) of the $(1 \times 1)$ unit cell, resulting in the weights $W_{m \mathbf{K}}\left(\mathbf{k}_{i}\right)$. The BandUP code deals with wave functions expressed in the plane-wave basis set. Thus we perform calculations with the VASP code $[44,45]$ based on the plane-wave basis, employing the projector-augmented wave (PAW) method [46,47]. The exchange correlation was calculated within the GGA [28], like in the LCPAO method. The energy cutoff was fixed at $350 \mathrm{eV}$ and a $6 \times 4 \times 1$ Monkhorst-Pack grid of $\mathbf{k}$ points was used. These calculations are notably more time consuming than the LCPAO calculations, thus we restrict ourselves to the case of NTCDA/Ag(111) only.

The band-structure calculations indicate that the deposition of a ML on top of the silver (111) surface influences the partly occupied SS in a qualitatively similar manner for different interfaces: the SS is shifted to a higher energy and transformed into the interface state (IS), due to the interaction of $\mathrm{Ag}$ with the molecular ML. In the case of the $\operatorname{Ag}(100)$ surface, the unoccupied Shockley resonance (SR) is also up-shifted in energy, which changes its character to a state residing in the projected band gap. The magnitude of the upshift depends on the factors, which affect the SS (or SR) properties mainly by steepening the surface potential to different extents. These are the type and relative density of molecules and adsorption geometry $[9,12,13,16-18]$. The calculated IS energies for all the interfaces under study are listed in Table I. One can see that the obtained values fall in the error range of the experimental results. Such a good agreement demonstrates the efficiency of the DFT in description of the metal-organic interfaces within the periodic slab geometry with a quite big number of atoms $(\sim 400)$ per unit cell.

In addition to the upshifted SS, the bare-surface electronic structure is filled up by weakly dispersive states related to the frontier orbitals of the molecular ML. These states are localized in the close vicinity of the monolayer [see Figs. 2(a)-(d)], and 
(a)

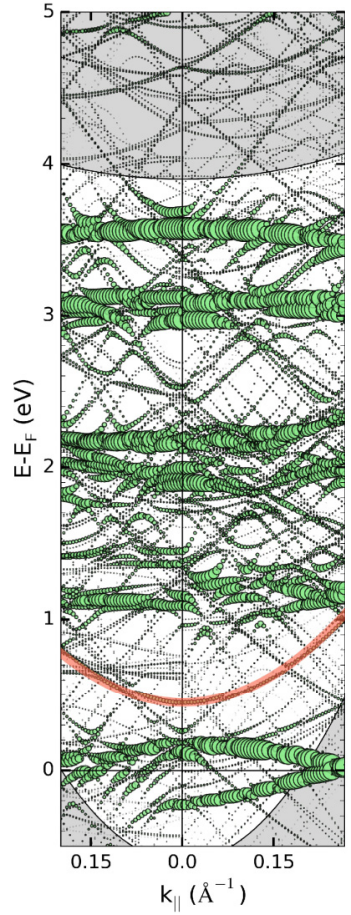

(b)

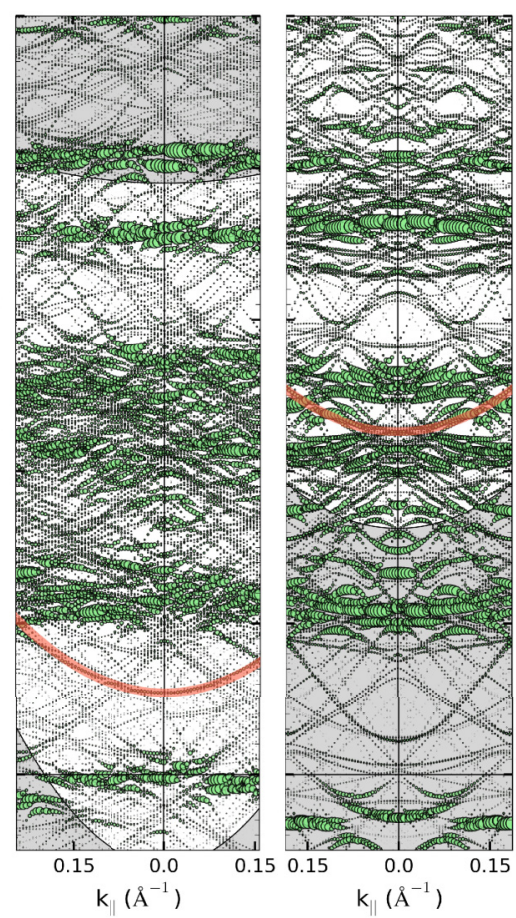

(d)

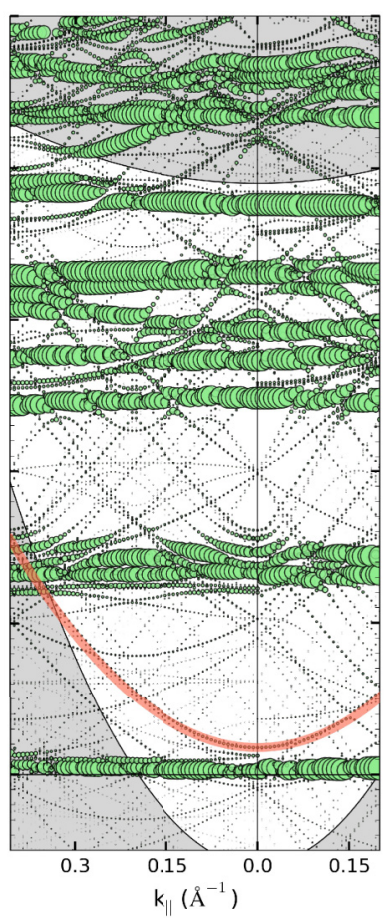

(e)

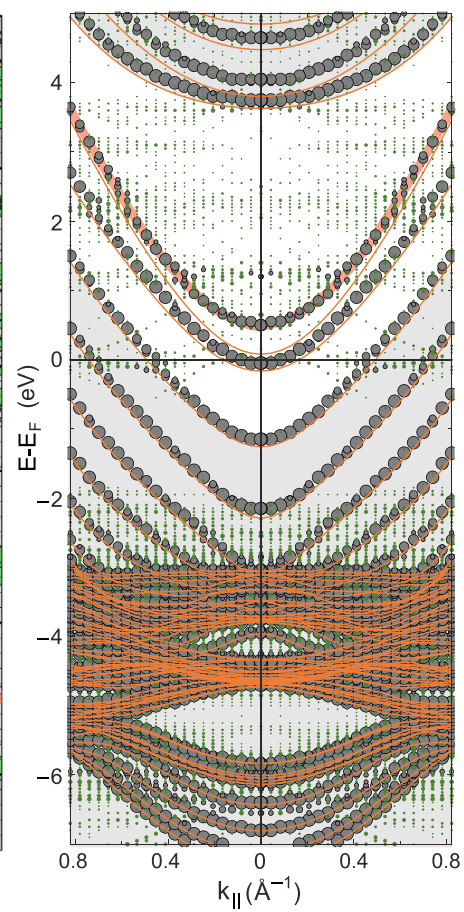

FIG. 2. (Color online) Band structure of (a) NTCDA/Ag(111) in the relaxed phase, (b) PTCDA/Ag(111), (c) PTCDA/Ag(100), and (d) PFP/Ag(111). The width of the fat bands reflects the extent of their localization in the vicinity of the molecular ML. The pink curves highlight the interface state derived from the Shockley surface state [or Shockley resonance in the case of $\operatorname{Ag}(100)$ ] of the respective bare silver surface. The gray area indicates the projection of bulk states onto the surface Brillouin zone. (e) Unfolded band structure of NTCDA/Ag(111) (solid circles) in comparison with that of the bare $\operatorname{Ag}(111)$ surface (orange lines). The size of the circle reflects the weight $W_{m \mathbf{K}}\left(\mathbf{k}_{i}\right)$ (varying from 0 to 1) of the state in the unfolded BZ, while its color discriminates the states with $W_{m \mathbf{K}}\left(\mathbf{k}_{i}\right) \geqslant 0.1$ (gray) from those with $W_{m \mathbf{K}}\left(\mathbf{k}_{i}\right)<0.1$ (green). The "green-colored" states can be attributed to the molecular-monolayer states highlighted in (a)-(d).

on the energy scale some of them lie below the IS. Therefore the molecular states are thought to have an effect on electron dynamics in the IS.

In the unfolded band structure [see Fig. 2(e)], one can see a clearer picture of how the deposition of an NTCDA ML modifies the electronic structure of the bare $\mathrm{Ag}(111)$ surface. Below $-3 \mathrm{eV}$ one can see a set of $d$ bands, which are not affected by the molecular ML. At higher energies the bulk-derived $s$ - $p$ bands forming the gap at the $\bar{\Gamma}$ point at $-1.2<E<3.6 \mathrm{eV}$ also have practically the same energies and dispersion for surfaces with and without NTCDA. The main effect of the NTCDA ML consists of the transformation of the bonding and antibonding surface states (at -0.17 and $+0.08 \mathrm{eV})$ of the bare ten-layer $\mathrm{Ag}(111)$ slab into the SS of the clean side of the slab (at $-0.06 \mathrm{eV}$ ) and into the IS of the side with NTCDA at $E_{\mathrm{IS}}=0.5 \mathrm{eV}$. The latter value agrees with the result of LCPAO calculations $E_{\mathrm{IS}}=0.4 \mathrm{eV}$. The molecular states of NTCDA are unfolded to different points of the UBZ with small weights, thus forming a weak background. Note that the spectral weight of the molecularderived states (MSs) are quite small, hence one could expect them to produce a rather minor contribution to the decay processes. This question will be addressed in more detail later in Sec. III C.

TABLE I. Experimental and theoretical values of the IS energy $E_{\mathrm{IS}}$ (in eV) and lifetimes $\tau_{\mathrm{IS}}$ (in fs). Theoretical values of $\tau_{\mathrm{IS}}$ are given for the $E$-shifted/ $V$-shifted scheme, accounting for inelastic electron-electron scattering only.

\begin{tabular}{|c|c|c|c|c|c|}
\hline & & PTCDA/Ag(111) & PTCDA/Ag(100) & NTCDA/Ag(111) & PFP/Ag(111) \\
\hline \multirow[t]{2}{*}{$E_{\mathrm{IS}}$} & Experiment & $0.57 \pm 0.02^{\mathrm{a}}$ & $2.25 \pm 0.03^{\mathrm{b}}$ & $0.38 \pm 0.02^{\mathrm{a}}$ & $0.1-0.2^{\mathrm{c}}$ \\
\hline & Theory & 0.55 & 2.26 & 0.40 & 0.17 \\
\hline \multirow[t]{2}{*}{$\tau_{\mathrm{IS}}$} & Experiment & $53 \pm 3^{a}$ & $3 \leqslant \tau \leqslant 18^{\mathrm{b}}$ & $115 \pm 10^{\mathrm{a}}$ & - \\
\hline & Theory & $110 / 270$ & $-/ 24$ & $250 / 500$ & $1280 / 1850$ \\
\hline
\end{tabular}

\footnotetext{
${ }^{\mathrm{a}}$ From Ref. [16].

${ }^{\mathrm{b}}$ From Ref. [18].

${ }^{c}$ From Ref. [48].
} 


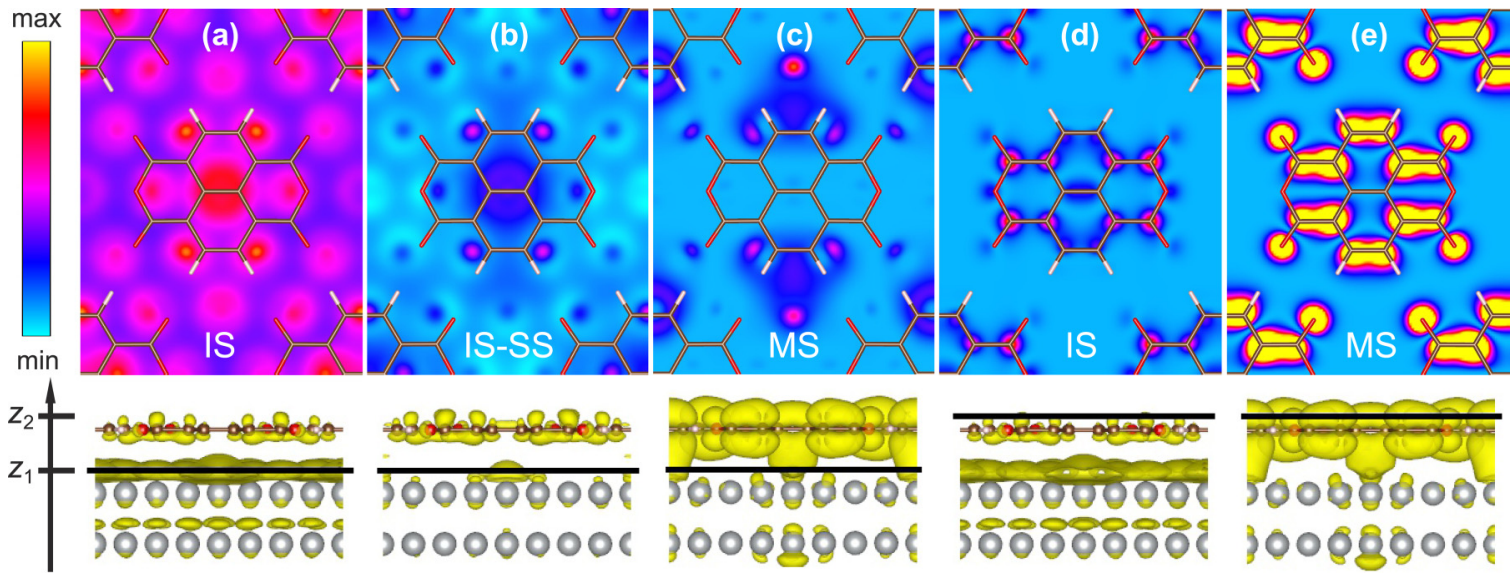

FIG. 3. (Color online) Charge density distribution $|\Psi(x, y, z)|^{2}$ of the interface state (IS) (a) and (d), the former-LUMO molecular state (MS) (c) and (e), and the difference between the charge-density distributions of the IS and the bare-surface surface state (SS) (b) on NTCDA/Ag(111). Top panels show the color maps of the corresponding $|\Psi(x, y, z)|^{2}$ in the $x-y$ plane parallel to the surface with the $z$ coordinate fixed at $z_{1}$ (a)-(c) and $z_{2}$ (d) and (e) (see the vertical dashed lines in Fig. 4). Lower panels represent the side view of the respective charge-density isosurfaces with black lines indicating the position of the $x-y$ plane of the color maps.

Now we turn from the interface band structure to the charge density distribution $|\Psi(x, y, z)|^{2}$ of the states of our interest. We address the question of how the IS differs from the SS in this aspect. Figure 3(a) shows the color map of $|\Psi(x, y, z)|^{2}$ of the IS at $\bar{\Gamma}$ as a function of $x$ and $y$ at the fixed $z=z_{1}$ right above the silver surface. This $z$ coordinate defines the plane parallel to the surface, where the difference of the IS and SS charge density distributions has a maximum over the whole region, where the overlapping between the IS and the bulk states dominates (see Fig. 4). As seen in Fig. 3(b), even in this plane the charge density distribution of the IS only slightly differs from the SS distribution [Fig 3(b)] mainly around the center of the molecule residing in the on-top position. Since it is consistent with the behavior of the interface potential in this plane, such a small difference visualizes the fact that the IS effective mass is nearly the same as that of the SS. The charge density distribution of the MS (former LUMO) in the plane with $z=z_{1}$ is of the same order of magnitude as the mentioned difference and has noticeable values [see Fig. 3(c)], where the IS is almost indistinguishable from the SS. In the IS distribution, notable features appear only in the vicinity of the molecular monolayer [Fig. 3(d)]. However, they do not

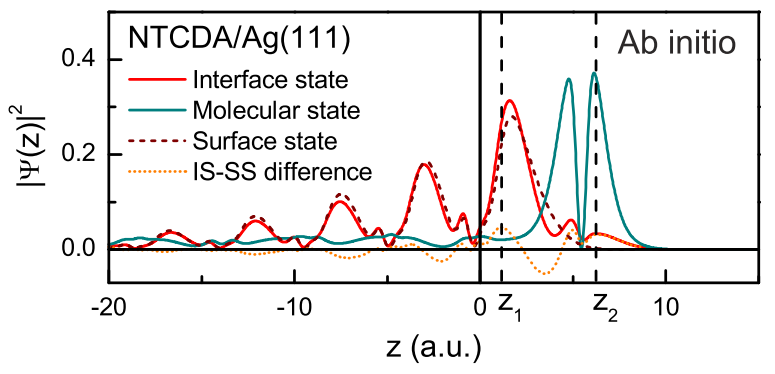

FIG. 4. (Color online) Calculated ab initio charge density distribution (averaged over the spatial coordinates $x y$ within the unit cell) of the interface and molecular states at the $\bar{\Gamma}$ point as functions of $z$ in comparison with that of the surface state of the bare $\operatorname{Ag}(111)$ surface. The difference between the interface state and surface-state charge-density distribution is shown as well. influence the rate of decay of electrons from the IS into the bulk. The molecular state is strongly localized on the atoms of NTCDA, and in the plane parallel to the molecular ML with $z=z_{2}$ [see Figs. 3(e) and 4] its charge density distribution resembles the LUMO of an isolated NTCDA.

\section{LIFETIMES OF ELECTRONS IN THE INTERFACE STATES}

In this study, the lifetimes of ISs, $\tau_{\mathrm{IS}}$, are calculated within the $G W$ formalism [21] of many-body perturbation theory. As far as a fully ab initio calculation of lifetimes for the interfaces under consideration is not feasible so far, one needs a model for the description of the electronic structure of these systems. Such a model can be based on the following propositions. First, as was mentioned above, the origin of the IS experimentally observed in the aforementioned interfaces is attributed to the upshifted SS (SR) of the bare surfaces (see, e.g., Refs. $[9,12,13,16-18])$. The properties of the resulting IS [the penetration into bulk, charge density distribution in the bulk and near-surface regions, energy-momentum $E_{\mathrm{IS}}(\mathbf{k})$ dispersion relation, etc.] are similar to those of the Shockleytype state residing in the projected band gap of the bare (111) surface [see Figs. 3(a), 3(b), and 4]. Second, as clearly seen in Fig. 2(e), the frontier orbitals of the molecular ML have rather small spectral weights in the unfolded electronic structure, and, as a consequence, for the IS the decay phase space is expected to be mainly provided by the projected Ag bulk states, which are practically not affected by the band folding caused by using the large interface unit cell instead of the $(1 \times 1)$ unit cell of the bare Ag surfaces. Third, since we consider the IS lifetime in the $\bar{\Gamma}$ point, excluding intraband transitions, the presence of the molecular ML changing the screening of the Coulomb interaction in the interface region should not have an effect on $\tau_{\text {IS }}$ mainly determined by overlapping between wave functions of the interface and bulk states. Thus, we reduce the problem to a study of electron lifetimes for a silverlike surface with a surface state modified in a way to reproduce the energy and dispersion of the considered IS. 
The mentioned simplifications allow us to build the description of the decay of IS electrons on the basis of the onedimensional (1D) pseudopotential model [22,23,49,50], which proved useful for the accurate description of single-particle and collective excitations on metal surfaces. This model allowed theoreticians to study the decay of electrons and holes in surface states [49-54], image potential states [22,23,56-61], and resonances [62] on clean metal surfaces, as well as in quantum-well states in ultrathin layers [55], corrals [63] on $\mathrm{Cu}(111)$, nano-islands [64,65], and on adsorbed atoms [66]. The main idea of this model is to take into account the variation of the potential and charge density distribution in the direction perpendicular to the surface only. In the plane parallel to the surface, the potential is assumed to be constant. However, the in-plane variations, which present in the real crystal potential, are considered within the effective mass method, allowing one to restore the energy-momentum dispersion relation $E(\mathbf{k})$ of the resulting states. Parameters of the model are fitted to reproduce the experimentally measured key features of the surface band structure: the energies and dispersion relations of the edges of the projected band gap, the surface state, and the first image-potential state. The calculations of lifetimes within this model generally yield results in reasonable agreement with experimental results and reproduce the observed trends, e.g., the dependence of the decay rate of image potential state on the wave vector [60] or with temperature [61].

\section{A. $G W$ approximation}

The method of lifetime calculations in the $G W$ approximation using a 1D pseudopotential is described in detail elsewhere [20], and here we give just a brief overview, indispensable for understanding of the further discussion of the results. In the one-shot realization of this approximation, which we use in our study, the self-energy is represented by the first term of its diagrammatic expansion in the screened Coulomb interaction as $\Sigma=i G_{0} W_{0}$, where the Green function $G_{0}$ is built on the single-particle states $\left\{\Psi_{\mathbf{k} i}\left(z, \mathbf{r}_{\|}\right)=\varphi_{i}(z) e^{i \mathbf{k} \mathbf{r}_{\|}}, E_{\mathbf{k} i}=\epsilon_{i}+k^{2} /\left(2 m_{i}^{*}\right)\right\}, \quad$ resulting from the solution of the one-dimensional Schrödinger equation (atomic units are used in the article, unless otherwise mentioned)

$$
\left[-\frac{1}{2} \frac{d^{2}}{d z^{2}}+V(z)\right] \varphi_{i}(z)=\epsilon_{i} \varphi_{i}(z) .
$$

The screened Coulomb interaction $W_{0}$ is calculated within random-phase approximation. In Eq. (1), $V(z)$ is the one-dimensional pseudopotential, which is discussed further in Secs. III B and III C.

In the approximation used, the decay rate $\Gamma_{\mathrm{e}-\mathrm{e}}$ of the electronic state in a band $i$ with the wave vector $\mathbf{k}$ is obtained as the projection of the imaginary part of the self-energy operator onto this state:

$$
\begin{aligned}
\Gamma_{\mathrm{e}-\mathrm{e}}= & -2\left\langle\Psi_{\mathbf{k} i}|\operatorname{Im} \Sigma| \Psi_{\mathbf{k} i}\right\rangle \\
= & -2 \sum_{j} \int d z d z^{\prime} M_{i j}\left(z, z^{\prime}\right) \int \frac{d \mathbf{q}}{(2 \pi)^{2}}\left[1-f_{\mathbf{q} j}\right] \\
& \times \theta\left(E_{\mathbf{k} i}-E_{\mathbf{q} j}\right) \operatorname{Im} W_{0}\left(z, z^{\prime} ; \mathbf{k}-\mathbf{q}, E_{\mathbf{k} i}-E_{\mathbf{q} j}\right) .
\end{aligned}
$$

Here $f_{\mathbf{q} j}$ is the Fermi factor and $M_{i j}\left(z, z^{\prime}\right)=$ $\varphi_{i}(z) \varphi_{i}\left(z^{\prime}\right) \varphi_{j}(z) \varphi_{j}\left(z^{\prime}\right)$.

Thus, the many-body decay rate is determined by three main factors: (i) the phase space of the final states $(\mathbf{q} j)$, (ii) the overlap between the wave functions of the initial and final states, and (iii) the magnitude of the imaginary part of the screened Coulomb interaction $\operatorname{Im} W_{0}$. The latter is given in linear response theory by

$$
\begin{aligned}
W_{0}\left(z, z^{\prime} ; \mathbf{q}, \omega\right)= & v_{c}\left(z, z^{\prime} ; \mathbf{q}\right)+\int d z_{1} d z_{2} v_{c}\left(z, z_{1} ; \mathbf{q}\right) \\
& \times \chi\left(z_{1}, z_{2} ; \mathbf{q}, \omega\right) v_{c}\left(z_{2}, z^{\prime} ; \mathbf{q}\right),
\end{aligned}
$$

where $v_{c}\left(z, z^{\prime} ; \mathbf{q}\right)=2 \pi e^{-q\left|z-z^{\prime}\right|} / q$ is the two-dimensional (2D) Fourier transform of the bare Coulomb interaction, and $\chi$ is the density-density response function of interacting electrons, which is evaluated within the random phase approximation from the equation

$$
\begin{aligned}
\chi\left(z, z^{\prime} ; \mathbf{q}, \omega\right)= & \chi^{0}\left(z, z^{\prime} ; \mathbf{q}, \omega\right)+\int d z_{1} d z_{2} \chi^{0}\left(z, z_{1} ; \mathbf{q}, \omega\right) \\
& \times v_{c}\left(z_{1}, z_{2} ; \mathbf{q}\right) \chi\left(z_{2}, z^{\prime} ; \mathbf{q}, \omega\right) .
\end{aligned}
$$

Here $\chi^{0}\left(\mathbf{r}_{1}, \mathbf{r}_{2} ; \omega\right)$ is the density-density response function of a noninteracting electron system:

$$
\chi^{0}\left(z, z^{\prime} ; \mathbf{q}, \omega\right)=\sum_{i j} \int \frac{d \mathbf{k}}{(2 \pi)^{2}} \frac{\left(f_{\mathbf{k} j}-f_{\mathbf{k}+\mathbf{q} j}\right) M_{i j}\left(z, z^{\prime}\right)}{\omega+E_{\mathbf{k} j}-E_{\mathbf{k}+\mathbf{q} i}+i \eta},
$$

with $\eta$ being an infinitesimally small positive constant.

\section{B. Model of shifted surface state}

We start with the pseudopotential introduced in Refs. [22,23] and the set of parameters ensuring the proper description of the surface electronic structure of the bare Ag surfaces. Furthermore, we need to modify the model to reproduce the energy of the IS, which in our study is presented by an SS shifted to higher energies against a background of the unchanged projected band-gap edges.

A way to achieve the shift of the SS is to change the corresponding energies $\epsilon_{i}$ entering Eqs. (2)-(5) "by hand," while leaving the wave functions $\varphi_{i}(z)$ unchanged. Hereafter we refer to this as the E-shifted surface state scheme. In this case we do not change the overlap between the IS and the bulk states, while the phase space and, to a certain extent, the screened interaction are modified. Another way is to tune the parameters of the 1D potential in the near-surface region providing a shift of the surface-state energy towards higher values at the $\bar{\Gamma}$ point (see Fig. 5). This scheme will be referred to as the $V$-shifted surface state scheme. Here all the aforementioned factors that determine the inelastic decay are affected. In both schemes the energy of the $n=1$ imagepotential state and the surface barrier are held unchanged.

In Fig. 6 the starting-point 1D potential and the corresponding wave functions, as well as their counterparts at the largest energies considered, are shown. As seen in the figure, the shifted-SS penetration into the bulk becomes smaller upon modifying the potential, leading to a redistribution of the SS charge density into the vacuum side. This redistribution to a certain extent reflects the situation observed in $a b$ initio calculations, where the charge of the interface state outside 

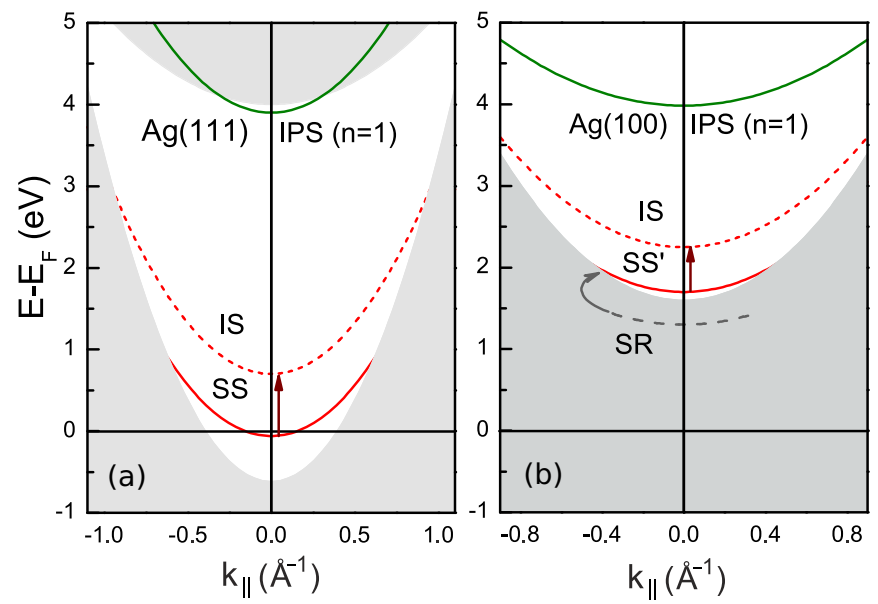

FIG. 5. (Color online) Sketch of the scheme realized in the model of the $V$-shifted surface state for $\mathrm{Ag}(111)$ (left) and $\mathrm{Ag}(100)$ (right). The SS is shifted towards higher energies by modifying the 1D pseudopotential of the bare $\mathrm{Ag}$ surfaces within the near-surface region. In the case of $\mathrm{Ag}(100)$, we start from the Shockley resonance (SR).

the metal is slightly larger than that of the surface state (see Fig. 4).

On the one hand, the $V$-shifted scheme looks more consistent, because the energies $\epsilon_{i}$ and wave functions $\varphi_{i}(z)$ are the solutions of the same Schrödinger equation (1). However, as follows from Fig. 4, despite the aforementioned redistribution on the vacuum side the penetration of the wave function into the metal (and, consequently, overlapping with the bulk states), remains similar to the case of the bare surface. This means that the $E$-shifted SS scheme is valid and can be more suitable in the lifetime description.

As an advantage, both developed schemes allow us to get $\Gamma_{\mathrm{e}-\mathrm{e}}$ as a function of the IS energy, instead of specific calculations for a given IS energy. The decay rate of the $V$-shifted SS electrons at $\bar{\Gamma}$ as a function of the SS(IS) energy is shown in Fig. 7(top) for the $\mathrm{Ag}(111)$ surface. For example, for the experimental values of the IS energy observed at $300 \mathrm{~K}$ for the ordered NTCDA and PTCDA monolayers on Ag(111) (see Table I) the decay rate $\Gamma_{\mathrm{e}-\mathrm{e}}=1.3 \mathrm{meV}(\tau \sim 0.5 \mathrm{ps})$ and $2.4 \mathrm{meV}$ ( $\tau \sim 0.3 \mathrm{ps}$ ), respectively. Such a difference between the indicated values of $\Gamma_{\text {e-e }}$ is caused by the fact that for the higher IS energy a larger decay phase space formed by the bulk states is available. However, the difference could be bigger, if the aforementioned overlap between the $\mathrm{SS}$ (IS) and the bulk states did not decrease with the increasing SS(IS) energy. Calculations performed with the unmodified bare-surface 1D potential but with changed SS(IS) energies (the $E$-shifted SS scheme) clearly show how the decreasing overlap reduces the gain in the decay phase space [see dashed line in Fig. 7(top)]. In this case, for the NTCDA and PTCDA monolayers on $\operatorname{Ag}(111)$ the decay rates are $\Gamma=2.6 \mathrm{meV}(\tau \sim 0.3 \mathrm{ps})$ and $6.0 \mathrm{meV}$ ( $\tau \sim 0.1 \mathrm{ps}$ ), respectively. The absolute values of the lifetimes found within both schemes for the interfaces are substantially longer than those experimentally observed: $\tau_{\mathrm{NTCDA} / \mathrm{Ag}(111)}^{\mathrm{IS}}=115 \pm 10 \mathrm{fs}$ and $\tau_{\mathrm{PTCDA} / \mathrm{Ag}(111)}^{\mathrm{IS}}=53 \pm 3 \mathrm{fs}$ at $300 \mathrm{~K}$ and $\tau_{\mathrm{NTCDA} / \operatorname{ISg}(111)}^{\mathrm{IS}}=43 \mathrm{fs}$ and $\tau_{\mathrm{PTCDA} / \operatorname{Ag}(111)}^{\mathrm{IS}}=26 \mathrm{fs}$

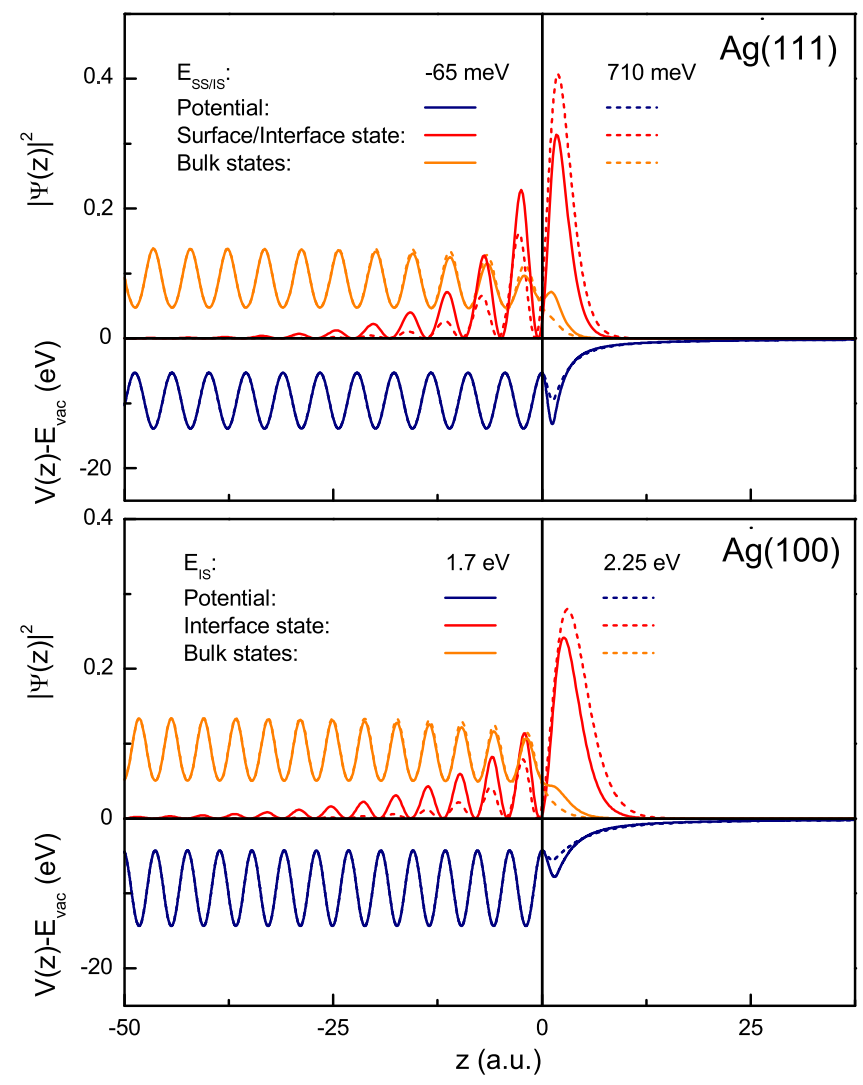

FIG. 6. (Color online) One-dimensional potential and respective wave functions for the surface (interface) states and bulk states in the case of the $\operatorname{Ag}(111)$ and $\operatorname{Ag}(100)$ surfaces at different energies of the shifted surface state $\left(E_{\mathrm{SS}(\mathrm{IS})}\right)$. The bulk charge density was normalized to fit the same scale as SS(IS).

at $90 \mathrm{~K}$ as reported in Ref. [16]. However, their ratio is very close to its experimental counterpart. Moreover, the presented results are in a nice accordance with the trend of the unusual increase of the lifetimes with rising temperature [see Fig. 7(top)]. Actually, at a higher temperature the absorption distance becomes larger and, as a consequence, due to the weakened interaction between the molecular ML and metal substrate, the IS energy gets smaller [16], thus providing the smaller decay rate.

For the PFP/Ag(111) interface, no experimental data are available on the IS lifetime. Our calculations yield a very small inelastic decay rate $\sim 0.5 \mathrm{meV}$, corresponding to the lifetime of 1-2 ps due to the low energy of the IS, and hence small phase space for the inelastic decay. However, this value should be sufficiently shortened by elastic decay channels, e.g., electron-defect scattering.

In the case of the $\operatorname{Ag}(100)$ surface, the decay rate of the surface resonance (SR) cannot be calculated within the present framework. However, when the SR is pushed into the band gap, it becomes the IS. The decay rate of the latter decreases with its energy [see Fig. 7(bottom)] in spite of the growing decay phase space. Such a decrease is caused by the rapid reduction of the IS-bulk states overlap (see Fig. 6). For the IS energy that corresponds to the PTCDA/Ag(100) interface, the decay rate is $27 \mathrm{meV}$. The corresponding lifetime $\tau=24 \mathrm{fs}$ is close to the upper limit of 18 fs found experimentally in Ref. [18]. Note 

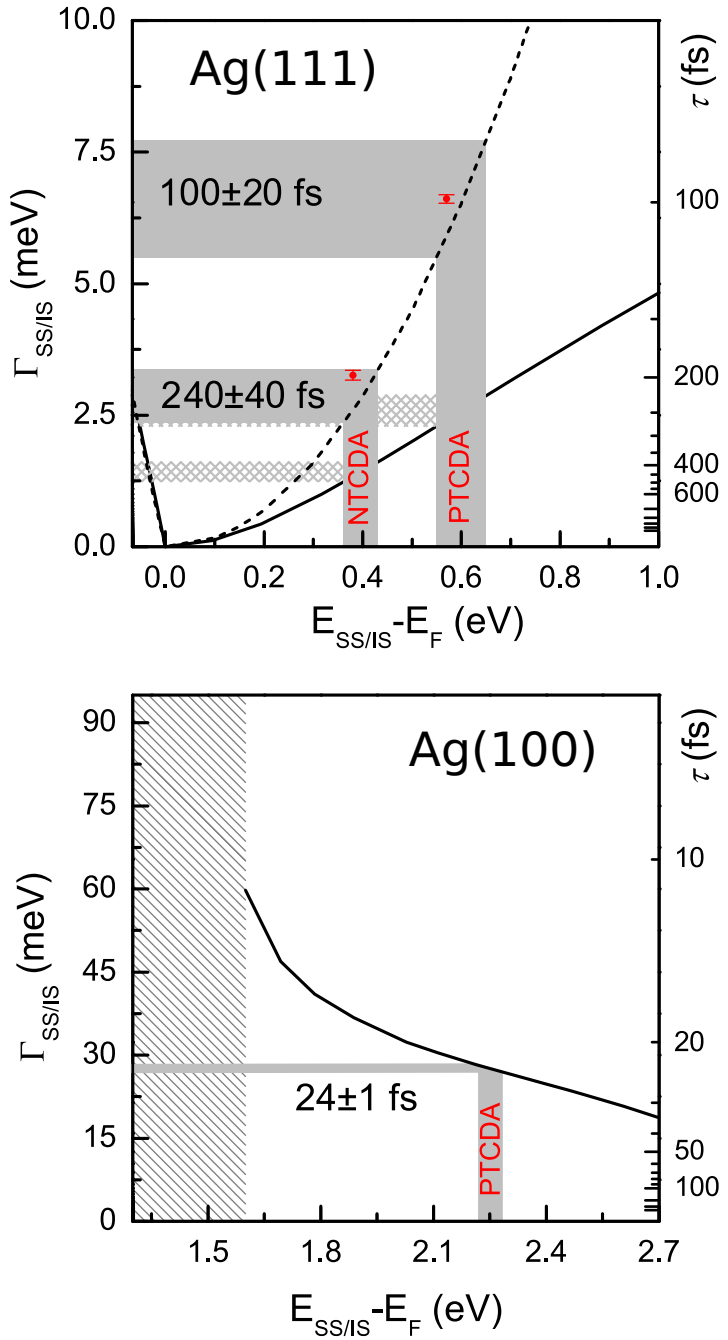

FIG. 7. (Color online) Calculated decay rate $\Gamma$ of the shifted surface state as a function of its energy. Solid (dashed) lines correspond to the $V$-(E-)shifted scheme, respectively. Light-gray stripes cover the energy intervals, which correspond to the experimental values of the IS energy, including its variations with temperature. In the case of $\operatorname{Ag}(111)$, red points show the decay rate as obtained within the $E$-shifted scheme with taking into account the contribution of the former LUMO. Error bars here are caused by changing the former LUMO energy (see Fig. 2) within the $\pm 50 \mathrm{meV}$ interval. In the case of $\mathrm{Ag}(100)$, the shaded area indicates the projected continuum of bulk states.

that we consider here the $V$-shifted SS scheme only, since we cannot extrapolate the resonancelike wave function of the bare $\mathrm{Ag}(100)$ surface to the projected band gap energy region.

\section{Monolayer pseudopotential}

In this section we make an attempt to take into account a possible contribution coming from the MSs lying energetically below the IS, in particular, the former LUMO in the $\operatorname{Ag}(111)$ based interfaces. These states are highlighted in Figs. 2(a)-2(d) by the fat bands and presented by green circles in Fig. 2(e). For this purpose, the energies and localizations of these states known from our ab initio calculations (Fig. 4) should be reproduced. To achieve this goal, we developed a pseudopotential

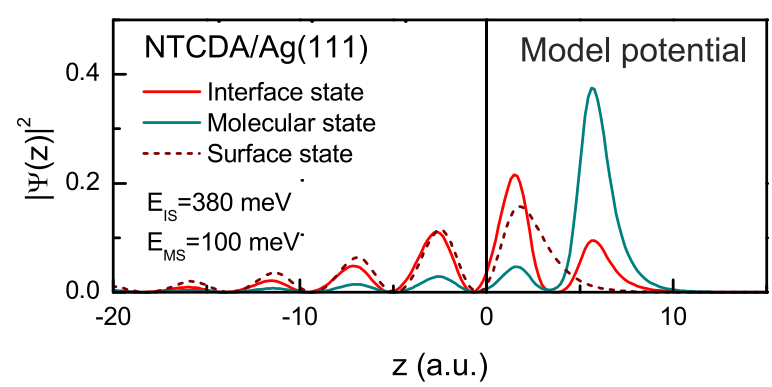

FIG. 8. (Color online) Charge density distribution of the interface and molecular states as compared with that of the surface state of the bare $\operatorname{Ag}(111)$ surface, calculated within the ML-pseudopotential model (see the text).

that models the molecular ML as a quantum well and contains a barrier, separating the ML from the bulk $\mathrm{Ag}(111)$. We refer to this model as ML potential. This model allows us to get the shifted SS and the desired MS with quite close energies, reproducing the $a b$ initio values, as well as the localization of the IS and MS in the interface region and at the molecular layer, respectively, with the overlapping $\int\left|\varphi_{\mathrm{IS}}(z)\right|^{2}\left|\varphi_{\mathrm{MS}}(z)\right|^{2} d z$ as obtained from the $a b$ initio calculations.

In Fig. 8 we plot the obtained model wave functions. Note, that in the calculation of lifetimes we have replaced the wave function of MS $\varphi_{\mathrm{MS}}(z)$ by $\varphi_{\mathrm{MS}}(z) / \sqrt{N}$ in order to take into account the unfolding onto the $(1 \times 1) \operatorname{Ag}(111) \mathrm{BZ}$. Here $N$ is the number of atoms in the $\mathrm{Ag}(111)$ surface layer unit cell of the interface: $N=33$ and 24 for PTCDA and NTCDA, respectively. The resulting contribution accounting for transitions from the $\mathrm{SS}$ (IS) to the MS was estimated to be $\sim 0.3-0.7 \mathrm{meV}$. Figure 7 (top) shows the quite moderate effect of this contribution being added to the decay rate of the $E$-shifted SS scheme (red dots in the figure). By error bars we demonstrate how variations in the MS energy $( \pm 50 \mathrm{meV})$ can affect the resulting $\Gamma$.

As one can see, the calculated lifetimes are generally longer than the experimental ones. On the one hand, this is a typical picture, because there are usually some other decay channels, which are not taken into account in our model consideration. On the other hand, the difference between theoretical and experimental results may be caused by inaccurate description of the charge density distribution along the $z$ axis. As follows from our $a b$ initio calculations, in the interfaces under study the presence of the molecular ML causes a redistribution of not only the SS charge density in the vicinity of the ML plane, as we show above, but also the bulk-states charge density. This simultaneous redistribution can produce an additional overlap between the IS and the bulk states, making the IS lifetime shorter. In order to take it into account, one should modify the 1D pseudopotential in a way to properly describe its behavior within the ML region. Since it involves the bulk states and affects the image-potential tail of the 1D pseudopotential, it should be done consistently with a study of energies and lifetimes of image-potential states, which, in turn, need to be experimentally analyzed first. Besides, further experimental studies of IS lifetimes for the organic monolayers on top of the $\operatorname{Ag}(111)$ surface might shed light on peculiarities of this surface that provide a distinct decay process as compared to the $\operatorname{Ag}(110)$ surface. 


\section{CONCLUSIONS}

We have presented a theoretical study of lifetimes of interface states in metal-organic interfaces within the models based on the use of one-dimensional pseudopotentials. The reported results allowed us to address the question about the description and explanation of recent experimental data. We have demonstrated that the folding of the BZ of the Ag surface due to substantial enlarging of the surface unit cell upon the deposition of ordered molecular monolayers does not have a drastic effect on the phase space of final states for electron decay.

By means of the unfolding procedure we demonstrated that the $\mathrm{BZ}$ folding upon organic monolayer deposition plays a minor role in the phase space for electron decay. Actually, it introduces only a weak background to the band structure of the $(1 \times 1)$ unit cell, and hence weakly affects the lifetimes. The presence of the unoccupied molecular state below the IS gives a small contribution to the decay rate of the IS, while the lifetime is mostly determined by the change of the phase space of final states upon the energy shift of the IS.

In the case of PTCDA/Ag(100), the IS lifetime obtained in the $E$-shifted SS scheme agrees well with the experimental data, while for PTCDA and NTCDA at Ag(111) these model strongly overestimates the corresponding lifetimes of the IS. Being applied to these $\operatorname{Ag}(111)$ interfaces, the $E$-shifted SS scheme, which is based on the phase-space description, yields shorter lifetimes, but still quite long as compared with the experiment. However, our calculations provide the ratio $\tau_{\mathrm{NTCDA} / \operatorname{Ag}(111)}^{\mathrm{IS}} / \tau_{\mathrm{PTCDA} / \operatorname{Ag}(111)}^{\text {IS }}$ in agreement with the experiments, and explain the trend of the unusual increase of the IS lifetimes with rising temperature.

\section{ACKNOWLEDGMENTS}

This work is a project of the SFB 1083 "Structure and Dynamics of Internal Interfaces" funded by the Deutsche Forschungsgemeinschaft (DFG). We acknowledge partial support from the University of Basque Country UPV/EHU (IT-756-13), the Departamento de Educación del Gobierno Vasco, The Tomsk State University Academic D.I. Mendeleev Fund Program (Grant No. 8.1.05.2015), the Spanish Ministry of Economy and Competitiveness MINECO (Grant No. FIS2013-48286-C2-1-P), and Saint Petersburg State University (Project No. 11.50.202.2015).
[1] J. Hwang, A. Wan, and A. Kahn, Mater. Sci. Eng. R 64, 1 (2009).

[2] N. Koch, J. Phys. Condens. Matter 20, 184008 (2008).

[3] F. S. Tautz, Prog. Surf. Sci. 82, 479 (2007).

[4] P. Szymanski, S. Garrett-Roe, and C. B. Harris, Prog. Surf. Sci. 78, 1 (2005).

[5] M. Marks, A. Schöll, and U. Höfer, J. Electron. Spectrosc. Relat. Phenom. 195, 263 (2014).

[6] J. Zhao, M. Feng, D. B. Dougherty, H. Sun, and H. Petek, ACS Nano 8, 10988 (2014).

[7] N. Ilyas and O. L. A. Monti, Phys. Rev. B 90, 125435 (2014).

[8] R. Temirov, S. Soubatch, A. Luican, and F. S. Tautz, Nature (London) 444, 350 (2006).

[9] C. H. Schwalb, S. Sachs, M. Marks, A. Schöll, F. Reinert, E. Umbach, and U. Höfer, Phys. Rev. Lett. 101, 146801 (2008).

[10] A. Yang, S. T. Shipman, S. Garrett-Roe, J. Johns, M. Strader, P. Szymanski, E. Muller, and C. Harris, J. Phys. Chem. C 112, 2506 (2008).

[11] A. Scheybal, K. Müller, R. Bertschinger, M. Wahl, A. Bendounan, P. Aebi, and T. A. Jung, Phys. Rev. B 79, 115406 (2009).

[12] M. S. Dyer and M. Persson, New J. Phys. 12, 063014 (2010).

[13] N. L. Zaitsev, I. A. Nechaev, and E. V. Chulkov, J. Exp. Theor. Phys. 110, 114 (2010).

[14] S. Sachs, C. H. Schwalb, M. Marks, A. Schöll, F. Reinert, E. Umbach, and U. Höfer, J. Chem. Phys. 131, 144701 (2009).

[15] C. H. Schwalb, M. Marks, S. Sachs, A. Schöll, F. Reinert, E. Umbach, and U. Höfer, Eur. J. Phys. 75, 23 (2010).

[16] M. Marks, N. L. Zaitsev, B. Schmidt, C. H. Schwalb, A. Schöll, I. A. Nechaev, P. M. Echenique, E. V. Chulkov, and U. Höfer, Phys. Rev. B 84, 081301(R) (2011).

[17] N. L. Zaitsev, I. A. Nechaev, P. M. Echenique, and E. V. Chulkov, Phys. Rev. B 85, 115301 (2012).
[18] M. C. E. Galbraith, M. Marks, R. Tonner, and U. Höfer, J. Phys. Chem. Lett. 5, 50 (2014).

[19] B. W. Caplins, D. E. Suich, A. J. Shearer, and C. B. Harris, J. Phys. Chem. Lett. 5, 1679 (2014).

[20] P. M. Echenique, R. Berndt, E. V. Chulkov, T. H. Fauster, A. Goldmann, and U. Höfer, Surf. Sci. Rep. 52, 219 (2004).

[21] L. Hedin, Phys. Rev. 139, A796 (1965).

[22] E. V. Chulkov, V. M. Silkin, and P. M. Echenique, Surf. Sci. 391, L1217 (1997).

[23] E. V. Chulkov, V. M. Silkin, and P. M. Echenique, Surf. Sci. 437, 330 (1999).

[24] http://openmx-square.org.

[25] T. Ozaki, Phys. Rev. B 67, 155108 (2003).

[26] T. Ozaki and H. Kino, Phys. Rev. B 69, 195113 (2004).

[27] T. Ozaki and H. Kino, Phys. Rev. B 72, 045121 (2005).

[28] J. P. Perdew, K. Burke, and M. Ernzerhof, Phys. Rev. Lett. 77, 3865 (1996).

[29] N. Troullier and J. L. Martins, Phys. Rev. B 43, 1993 (1991).

[30] U. Stahl, D. Gador, A. Soukopp, R. Fink, and E. Umbach, Surf. Sci. 414, 423 (1998).

[31] R. Fink, D. Gador, U. Stahl, Y. Zou, and E. Umbach, Phys. Rev. B 60, 2818 (1999).

[32] C. Stadler, S. Hansen, A. Schöll, T.-L. Lee, J. Zegenhagen, C. Kumpf, and E. Umbach, New J. Phys. 9, 50 (2007).

[33] C. R. Braatz, T. Esat, C. Wagner, R. Temirov, F. S. Tautz, and P. Jakob, Surf. Sci. 643, 98 (2016).

[34] K. Glöckler, C. Seidel, A. Soukopp, M. Sokolowski, E. Umbach, M. Böhringer, R. Berndt, and W.-D. Schneider, Surf. Sci. 405, 1 (1998).

[35] L. Kilian, E. Umbach, and M. Sokolowski, Surf. Sci. 573, 359 (2004). 
[36] A. Hauschild, R. Temirov, S. Soubatch, O. Bauer, A. Schöll, B. C. C. Cowie, T.-L. Lee, F. S. Tautz, and M. Sokolowski, Phys. Rev. B 81, 125432 (2010).

[37] S. L. Wong, H. Huang, Y. L. Huang, Y. Z. Wang, X. Y. Gao, T. Suzuki, W. Chen, and A. T. S. Wee, J. Phys. Chem. C 114, 9356 (2010).

[38] S. Duhm, S. Hosoumi, I. Salzmann, A. Gerlach, M. Oehzelt, B. Wedl, T.-L. Lee, F. Schreiber, N. Koch, N. Ueno, and S. Kera, Phys. Rev. B 81, 045418 (2010).

[39] J. Götzen, C. H. Schwalb, C. Schmidt, G. Mette, M. Marks, U. Höfer, and G. Witte, Langmuir 27, 993 (2011).

[40] BandUP: Band unfolding code for plane-wave based calculations, www.ifm.liu.se/theomod/compphys/band-unfolding.

[41] P. V. C. Medeiros, S. Stafström, and J. Björk, Phys. Rev. B 89, 041407(R) (2014).

[42] P. V. C. Medeiros, S. S. Tsirkin, S. Stafström, and J. Björk, Phys. Rev. B. 91, 041116(R) (2015).

[43] V. Popescu and A. Zunger, Phys. Rev. B 85, 085201 (2012).

[44] G. Kresse and J. Hafner, Phys. Rev. B 48, 13115 (1993).

[45] G. Kresse and J. Furthmüller, Comput. Mater. Sci. 6, 15 (1996).

[46] P. E. Blöchl, Phys. Rev. B 50, 17953 (1994).

[47] G. Kresse and D. Joubert, Phys. Rev. B 59, 1758 (1999).

[48] M. C. E. Galbraith, M. Sc. thesis, Philipps-Universität Marburg, 2012.

[49] J. Kliewer, R. Berndt, E. V. Chulkov, V. M. Silkin, P. M. Echenique, and S. Crampin, Science 288, 1399 (2000).

[50] L. Vitali, P. Wahl, M. A. Schnider, K. Kern, V. M. Silkin, E. V. Chulkov, and P. M. Echenique, Surf. Sci. Lett. 523, L47 (2003).

[51] S. Link, H. A. Dür, G. Bihlmayer, S. Blügel, W. Eberhardt, E. V. Chulkov, V. M. Silkin, and P. M. Echenique, Phys. Rev. B 63, 115420 (2001).
[52] A. Schäfer, I. L. Shumay, M. Wiets, M. Weinelt, Th. Fauster, E. V. Chulkov, V. M. Silkin, and P. M. Echenique, Phys. Rev. B 61, 13159 (2000).

[53] E. V. Chulkov, V. M. Silkin, and M. Machado, Surf. Sci. 482485, 693 (2001).

[54] I. A. Nechaev, M. F. Jensen, E. D. L. Rienks, V. M. Silkin, P. M. Echenique, E. V. Chulkov, and Ph. Hofmann, Phys. Rev. B 80, 113402 (2009).

[55] E. V. Chulkov, J. Kliewer, R. Berndt, V. M. Silkin, B. Hellsing, S. Crampin, and P. M. Echenique, Phys. Rev. B 68, 195422 (2003).

[56] J. Osma, I. Sarría, E. V. Chulkov, J. M. Pitarke, and P. M. Echenique, Phys. Rev. B 59, 10591 (1999).

[57] I. Sarria, J. Osma, E. V. Chulkov, J. M. Pitarke, and P. M. Echenique, Phys. Rev. B 60, 11795 (1999).

[58] S. Crampin, Phys. Rev. Lett. 95, 046801 (2005).

[59] P. Saalfrank, M. Nest, I. Andrianov, T. Klamroth, D. Kroner, and S. Beyvers, J. Phys.: Condens. Matter 18, S1425 (2006).

[60] K. Schubert, A. Damm, S. V. Eremeev, M. Marks, M. Shibuta, W. Berthold, J. Güdde, A. G. Borisov, S. S. Tsirkin, E. V. Chulkov, and U. Höfer, Phys. Rev. B 85, 205431 (2012).

[61] S. S. Tsirkin, S. V. Eremeev, E. V. Chulkov, M. Marks, K. Schubert, J. Güdde, and U. Höfer, Phys. Rev. B 86, 085424 (2012).

[62] S. S. Tsirkin, A. G. Borisov, and E. V. Chulkov, Phys. Rev. B 88, 035449 (2013).

[63] S. Díaz-Tendero, F. E. Olsson, A. G. Borisov, and J.-P. Gauyacq, Phys. Rev. B 77, 205403 (2008).

[64] T. Hakala, M. J. Puska, A. G. Borisov, V. M. Silkin, N. Zabala, and E. V. Chulkov, Phys. Rev. B 75, 165419 (2007).

[65] E. Ogando, N. Zabala, E. V. Chulkov, and M. J. Puska, Phys. Rev. B 69, 153410 (2004).

[66] A. G. Borisov, J. P. Gauyacq, A. K. Kazansky, E. V. Chulkov, V. M. Silkin, and P. M. Echenique, Phys. Rev. Lett. 86, 488 (2001). 